\title{
The fractal dimension of variations of the solar magnetic field
}

\author{
G. S. Ivanov-Kholodny $\dagger$, E. I. Mogilevsky \\ and V. E. Chertoprud
}

Institute of Terrestrial Magnetism, Ionosphere and Radio Wave Propagation RAS, Troitsk of Moscow region, 142190, Russia IN 12345, USA email:gor@izmiran.rssi.ru

Analysis of time series of the solar magnetic field energy (SMFE) at various solar latitudes $\left(\varphi=-75^{\circ} \div+75^{\circ}\right)$ has been done. Higuchi's technique Higuchi(1988) to measure the fractal dimension (FD) of time series has been chosen and the daily values $B(\varphi, t)$ of the solar magnetic field on the source surface for 1960-1999 have been used. These values had been computed in IZMIRAN using method by Obridko et al. (1994) As a matter of fact, Higuchi's measurements of the FD of time series $X_{t}$ comes to calculation of the length of the curve $l(k) \sim<\left|X_{t+k}-X_{t}\right|>k^{-2}$ and estimate of the coefficient $D$ in equation $\lg l(k)=A-D \lg k$ for considered range of $k$. The dimensions $D(\varphi, K)$ of the time series $B(\varphi, t)^{2}$ have been computed for various time scales $0<k<K$ $\left([k]=[t]=1^{d}\right)$. It has been found that at $k>10-27$ the time series of SMFE form fractal with $D \approx 1.9$.

To study time variations of $\mathrm{FD}$, the additional estimates of values $D(\varphi, K, t)$ have been done on a running year interval $(t-182, t+182)$. It has been found out, that running estimates of the FD vary in time essentially. At $K<27$ both 11-year cycle prevailing at high latitudes and dominant at low latitudes quasi-biennial variations (QBV) are precisely traced in these changes. Applying the linear filtration according to Ivanonkholodny et al. (1992), it is possible to reveal QBV in $D(\varphi, K, t)$ practically in all range of latitudes. These are rather similar to the QBV of solar activity indices. Correlation coefficients between the QBV of $D(t)$ and of $F_{10.7}(t+\tau)$ are approximately equal 0.5 when $\tau$ is 6-8 months. An example of the QBV in solar activity index $\left(F_{10.7}\right)$ and in FD after a linear filtration is shown in fig. 1 . As well as in [3], the running year estimations of $F_{10.7}$ were used. Estimations of $D(\varphi, K, t)$ were determined for the latitude range $|\varphi|<40^{\circ}$ at $K=14\left(D_{14}\right)$ and $K=27\left(D_{27}\right)$. Numerals on graphs designate serial numbers of $F_{10.7}$ QBV maxima as well as the maxima appropriate to them in QBV for $D_{14}$ and $D_{27}$. Thus, solar QBV, shown almost in all indices of solar activity, are also present in fractal structure of the SMFE.

The following conclusions have been made. 1.The time series of the SMFE are a typical fractal set. 2. The running estimations of the fractal dimension are rather suitable to gain new information about QBV. 3. It is possible to conclude, that, QBV, found out in changes $\mathrm{FD}$, are related to $\mathrm{QBV}$ in indices of solar activity.

\section{Acknowledgements}

Authors are grateful to V.N. Obridko and B.D. Shelting for providing the time series of the solar magnetic field on the source surface. This work was supported by RFBR (project 04-02-16374a).

$\dagger$ Present address: Institute of Terrestrial Magnetism, Ionosphere and Radio Wave Propagation RAS, Troitsk, Russia. 

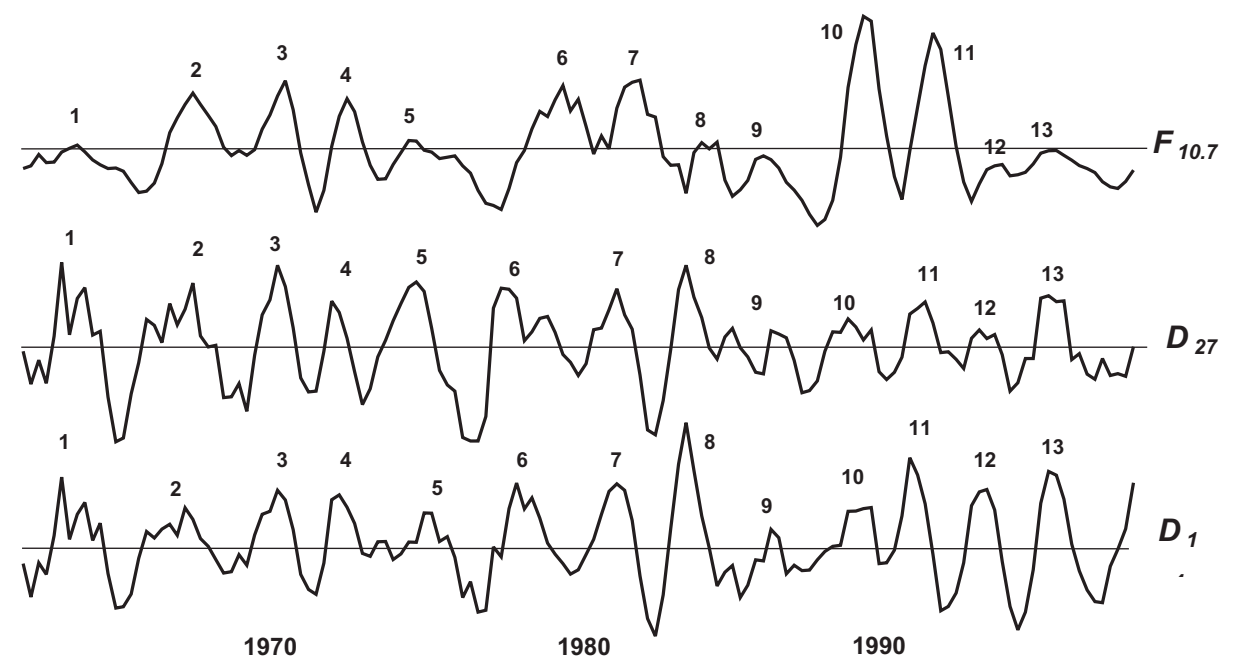

Figure 1. Quasi-biennial variations of both the solar activity $\left(F_{10.7}\right)$ and fractal dimension of the SMFE $\left(D_{14}\right.$ and $\left.D_{27}\right)$ after filtration.

\section{References}

Higuchi, T. 1988 Physica D 31, 225-245.

Ivanov-kholodny, G. S.\& Chertoprud, V. E. 1992 J. Astronom.Astrophys.Transect. 3, 81-84.

Obridko V. N. Harshiladze, A. F. and Shelting, B. D. 1999 In Ninth Intl Conf. on Numerical Methods in Fluid Dynamics (ed. Soubbaramayer \& J. P. Boujot). Lecture Notes in Physics, vol. 218, pp. 23-51. Springer. 